\title{
Evidence for F-theory
}

\section{Citation}

Vafa, Cumrun. 1996. "Evidence for F-Theory." Nuclear Physics B 469 (3): 403-15. https:// doi.org/10.1016/0550-3213(96)00172-1.

\section{Permanent link}

http://nrs.harvard.edu/urn-3:HUL.InstRepos:41385066

\section{Terms of Use}

This article was downloaded from Harvard University's DASH repository, and is made available under the terms and conditions applicable to Other Posted Material, as set forth at http:// nrs.harvard.edu/urn-3:HUL.InstRepos:dash.current.terms-of-use\#LAA

\section{Share Your Story}

The Harvard community has made this article openly available.

Please share how this access benefits you. Submit a story.

Accessibility 
HUTP-96/A004

hep-th/9602022

\title{
Evidence for F-Theory
}

\author{
Cumrun Vafa \\ Lyman Laboratory of Physics, Harvard University \\ Cambridge, MA 02138, USA
}

\begin{abstract}
We construct compact examples of D-manifolds for type IIB strings. The construction has a natural interpretation in terms of compactification of a 12 dimensional ' $\mathrm{F}$-theory'. We provide evidence for a more natural reformulation of type IIB theory in terms of Ftheory. Compactification of M-theory on a manifold $K$ which admits elliptic fibration is equivalent to compactification of F-theory on $K \times S^{1}$. A large class of $N=1$ theories in 6 dimensions are obtained by compactification of F-theory on Calabi-Yau threefolds. A class of phenomenologically promising compactifications of F-theory is on $\operatorname{Spin}(7)$ holonomy manifolds down to 4 dimensions. This may provide a concrete realization of Witten's proposal for solving the cosmological constant problem in four dimensions.
\end{abstract}

Feb. 1996 


\section{Introduction}

Discovery of string dualities has opened up a new era in our understanding of string theory. A global picture of dualities has not yet emerged and it seems that different dualities are most easily 'understood' from different views. One successful viewpoint has been the notion of M-theory [1]国国田国. Even though this has led to many insights into string dualities, there are some cases that appear less naturally in this formulation. For example to understand the $S L(2, \mathbf{Z})$ invariance of type IIB in ten dimensions we first have to compactify down to nine dimensions and compare with $T^{2}$ compactifications of M-theory [1] [6] where $S L(2, \mathbf{Z})$ gets interpreted as the symmetry of the torus. However, only in the limit where $T^{2}$ is of zero area do we obtain type IIB in ten dimensions. It would have been far more satisfactory if the $S L(2, \mathbf{Z})$ invariance of type IIB in ten dimension had a geometric meaning already in ten dimensions.

In this paper we face this question even more strongly in consideration of compactifications of type IIB string on D-manifolds [7]. So far it has been difficult to obtain compact examples of such manifolds- here we will find that if the p-branes are D-branes of different $(p, q)$ strings [1], one can construct compact examples. In arguing for the existence of these vacua a 12 dimensional viewpoint emerges. One can have two views about this 12 dimensional origin of type IIB: either it is an auxiliary manifold just useful for constructing vacua of string theory involving D-manifolds or it is more real. In support of the latter interpretation, which we call the 'F-Theory', we point out that this 12 dimensional viewpoint also solves another puzzle of type IIB strong/weak duality: The D-string, having a gauge field on its worldsheet has a critical dimension of 12 and actually lives in $(10,2)$ space and is related to ten dimensional type IIB theory by a null reduction [8]. The on shell physical states only carry ten dimensional momenta. However off-shell states may have more non-trivial dependence on the extra two coordinates and may make them more real.

As concrete examples we consider compactification of F-theory on $K 3$ which can also be interpreted as type IIB compactification on a D-manifold, $S^{2}$, where we have turned on 24 7-branes on $S^{2}$, and argue that it is dual to heterotic string compactification on $T^{2}$. We also consider compactifications of F-theory to 6,5 and 4 dimensions on Calabi-Yau, $G_{2}$ holonomy manifolds and $\operatorname{Spin}(7)$ holonomy manifolds. Upon compactification on a circle these would be related to M-theory compactifications on the same manifolds. The 6-dimensional compactification of F-theory lead to new type II vacua with $N=1$ supersymmetry with a number of tensor multiplets and gauge multiplets. Compactifications of 
F-theory on $\operatorname{Spin}(7)$ manifolds down to 4 dimensions apparently has no supersymmetry. However upon compactification to three dimensions it is related to a supersymmetric Mtheory. This may lead to a solution of the cosmological constant problem along the lines proposed by Witten [9].

\section{New Vacua for Type IIB Strings}

The bosonic content of the type IIB consists of $g_{\mu \nu}, B_{\mu \nu}^{A}, \phi$ from the NS-NS sector and $\tilde{\phi}, B_{\mu \nu}^{P}, A_{\mu \nu \alpha \beta}^{+}$from the R-R sector. Under the conjectured ten dimensional $S L(2, \mathbf{Z})$ strong/weak duality [10] the complex field $\tau=\tilde{\phi}+i \exp (-\phi)$ transforms in the same way as the modulus of a torus. The antisymmetric tensor fields $B^{A}$ and $B^{P}$ get exchanged and the metric $g$ and the four form potential $A^{+}$are invariant.

Let us consider vacuum solutions of type IIB for which $B^{A}=B^{P}=A^{+}=0$. We first consider solutions which leave an 8-dimensional Lorentz group invariant. Let $z$ denote as a complex coordinate the 9-10 direction. The relevant low energy lagrangian is

$$
S=\int \sqrt{g}\left(R+\frac{\partial \tau \partial \bar{\tau}}{\tau_{2}^{2}}\right)
$$

Then we are looking for solutions of low energy lagrangian where $\tau(z, \bar{z})$ and $g_{z \bar{z}}(z, \bar{z})$ vary over $z$ and the rest of the components of $g$ are flat. Moreover we look for solutions where half of the supersymmetries are preserved. This BPS condition implies that $\tau$ is only a function of $z$ (or $\bar{z}$ ). An example of this is the vacuum for a Dirichlet 7-brane, where the worldvolume of the 7-brane coincides with the uncompactified 8 dimensional spacetime. Let the 9-10 position of the 7-brane be at $z=0$. Since a 7 -brane is a magnetic charge for the $\tilde{\phi}$ field, it implies that as we circle $z=0$, we have $\tau \rightarrow \tau+1$. Together with the holomorphy of $\tau$ we learn that near $z=0$

$$
\tau(z) \sim \frac{1}{2 \pi i} \log (z)
$$

Near $z=0$ the above adiabatic description of the action breaks down. However that is precisely the regime where we can use perturbative string theory and we know that the above solution is consistent with having a Dirichlet 7-brane at the origin and so is an acceptable solution. However the above ansatz runs into infrared difficulty for large $z$. Viewed as a 'cosmic string' the energy per unit length of this solution diverges which means that the equation for gravity cannot be solved in a consistent way. This is exactly 
the same problem encountered and solved in the context of 'stringy cosmic strings' [11]. In fact the connection between 7-branes of type IIB and stringy cosmic strings has already been pointed out in [12]. In the case of stringy cosmic string one was considering a toroidal compactification which led to a complex moduli $\tau$. To construct finite energy solutions where $\tau$ depends holomoprhically on a complex parameter $z$ it was found that it is crucial to use the ambiguity of $\tau$ to allow $S L(2, \mathbf{Z})$ jumps in $\tau$.

Once we allow jumps up to an $S L(2, \mathbf{Z})$ we can construct a solution. To get a compact space we need to wrap the complex plane 24 times around the fundamental domain of $S L(2, \mathbf{Z})$. In the case considered in [11] this gives rise to the four dimensional $K 3 \mathrm{com}-$ pactification when we take into account the 'hidden' torus. In fact the existence of the hidden torus was crucial in [11] to argue for the existence of this solution. In particular in regions were $\tau_{2} \rightarrow \infty$ the fact that the total space, including the $T^{2}$, is not singular, was crucial in establishing the consistency of the solution. Here we can use the same solution as there, except that now we seem to have no 'hidden' torus! The analogy is so strong that we are led to search for a hidden torus and in fact in the next section we present evidence for its existence. However, in this section let us continue our discussion of the properties of the 8-dimensional solution we have just constructed.

To make the solution more concrete let us specify how $\tau$ depends on $z$. Consider the torus given by

$$
y^{2}=x^{3}+f^{8}(z) x+f^{12}(z)
$$

where $f^{n}$ are polynomials of degree $n$ in $z$. The above equation defines a torus as a function of $z$. The ratio of the degrees of the two polynomials is set by the condition that as $z \rightarrow \infty$ we get a non-singular torus. Moreover the total degree of each is set by the condition that we wrap the $z$-sphere 24 times around the complex moduli of $T^{2}$. To see this note that the points where the torus degenerates corresponds to where the discriminant of the cubic vanishes, i.e. when

$$
27\left(f^{8}\right)^{3}-4\left(f^{12}\right)^{2}=0
$$

which is a polynomial of degree 24, and thus has 24 solutions. Note that the number of independent variables in the above solution is 18: 9 coefficients from $f^{8}$ plus 13 coefficients from $f^{12}$, minus 3 from $S L(2, C)$ action on the $z$-plane minus 1 from the fact that scaling $f^{8} \rightarrow \lambda^{2} f^{8}$ and $f^{12} \rightarrow \lambda^{3} f^{12}$ does not change the torus. On the other hand, the solution in [11] allows for two further moduli: One for the size of the $S^{2}$ and the other for the size of $T^{2}$. For us, the size for $T^{2}$ is not dynamical so that is not a moduli. However the size of $S^{2}$ 
is observable. Note that we cannot turn on $B^{A}$ or $B^{P}$. Not even to set them to a constant, because we are using $S L(2, \mathbf{Z})$ transformations and they are not invariant under it. So they are frozen to be zero. Thus in particular the Kahler moduli of $S^{2}$ is a real parameter and is not complexified as is customary in more conventional string backgrounds. So the total moduli is 18 complex parameters describing the complex moduli of the elliptic fibration over $P^{1}$ and one real parameter describing the size of $S^{2}$. Note that we have managed to find a compact manifold where we have a condensation of D-branes. This is the first compact example of D-manifolds proposed in [7]. Note that unlike open strings where condensation of D-branes is very natural [13] [14], for closed strings the story was not so clear. In fact there was a kind of 'no-go theorem' which runs as follows: If we have a D-brane in a compact manifold then the flux of the D-brane charge has nowhere to go, and so we would run into inconsistencies. What has happened in the example constructed above is that we have used the non-abelian nature of the relative D-brane charges coming from different $(p, q)$ strings related to each other by $S L(2, \mathbf{Z})$ to avoid any inconsistencies.

Let us see how many gauge fields we will get in this compactification. The fact that we have twenty four 7-branes, and that each one comes with a $U(1)$ gauge field, might at first suggest that we have generically a $U(1)^{24}$. This however cannot be the case. The easiest way to see this is that the condensation of scalars which are in the adjoint of the $U(1)$ 's are responsible for changing the relative position of the 7-branes. However above we found that there are at most 18 relative positions (in the 9-10 plane) free to be changed. Thus we have $U(1)^{18}$ plus another $U(1)^{2}$ coming from the overall complex shift of all the 7-branes which are the graviphotons.

This reduction of the number of $U(1)$ gauge fields from a naive counting of the Dbranes deserves further comments. Even though we have twenty four 7-branes, they are not perturbative D-branes of a given string theory. More generally they are the perturbative D-branes of some $(p, q)$ string, because we have used $S L(2, \mathbf{Z})$ in constructing our solution. Near a region where we have a 7-brane of a $(p, q)$ theory we can use perturbative string description of the $(p, q)$ theory to study the structure of string spectrum. However in going from one $(p, q)$ string spectrum to another, we may be double counting a given state. In the case at hand it turns out that we can get at most 18 of the D-branes to be the 7-branes of a given theory. This is consistent with the fact that the other $U(1)$ 's are linear combinations of these $18 U(1)$ 's.

Note that in addition to the gauge fields, we have $A^{+} 4$-form. We can either take two component to be the volume element on $S^{2}$, leaving us with a 2 -form in 8 dimensions, 
or we can take it to be the uncompactified components which is dual to a 2 -form in 8 dimensions. Together with the fact that $A^{+}$has a self-dual field strength, we thus end up with one antisymmetric two form in eight dimensions. Putting all the spectra and the number of supersymmetries together we see that we have exactly the same spectrum as the toroidal compactification of the heterotic string from 10 to 8 dimensions. We conjecture that our solution is dual to it, where the role of the coupling of heterotic string is played by the size of $S^{2}$. Weak coupling heterotic string corresponds to small $S^{2}$ and large coupling corresponds to big $S^{2}$.

One simple check for this identification is the fact that the field strength of the antisymmetric field in 8 dimensions, $H$, in both cases satisfies

$$
d H=\frac{1}{2}\left[p_{1}(R)-p_{1}(F)\right]
$$

where $p_{1}(R)$ (resp. $p_{1}(F)$ ) refer to first pontryagin class of $R$ (resp. $F$ ). In the case at hand such a correction is present. To see this note that $H$ is the field strength dual to the $\mathrm{RR}$ gauge potential $A^{+}$in eight dimensions and view the 8 dimensional spacetime as the worldbrane of the 7-branes. Then as argued in [15] in the worldbrane of the D-branes there are such corrections (and we need exactly 24 7-branes to get the coefficient of $p_{1}(R)$ to come out correctly). That there are such corrections was deduced implicitly in [15]. At least for the $p_{1}(F)$ term a direct computation of this has been performed [16] [17] [18].

Let us give two additional arguments in favor of this conjectured duality 1 . Consider the compactification of M-theory on $K 3$ which are themselves $T^{2}$ fibration over $S^{2}$. Since compactification of M-theory on $T^{2}$ is equivalent to compactification of type IIB theory on $S^{1}$, to the extent that we can trust the adiabatic argument [19], we end up on type IIB side with a compactification on $S^{1} \times S^{2}$ where $\tau$ varies over $S^{2}$ as in the above. So we see that the compactification of the above solution on a further $S^{1}$ is on the same moduli as M-theory on $K 3$ which is also conjectured [20 to be on the same moduli as $T^{3}$ compactification of heterotic string.

The second argument is as follows: If we compactify further on $T^{2}$ and use $T$-duality once on each of the two circles of $T^{2}$ we end up again with type IIB, but now instead of twenty four 7-branes we have twenty four 5-branes. In fact it was shown locally [21] [7] that type IIB compactified on a D-manifold with 24 -banes is dual to type IIA compactified on $K 3$ which is itself conjectured to be dual to heterotic string on $T^{4}$ [10] [22]. Here we

1 The first one was pointed out to us by N. Seiberg. 
have managed to find a concrete global realization of this proposal[ ${ }^{2}$. Recall that in [7] the identification of the behaviour of type IIA compactification near an $A_{n-1}$ singularity of K3 with type IIB on $n$ symmetric 5-branes [21] coupled with strong/weak duality of type IIB relating this to $n$ coincident Dirichlet 5 -branes predicted the appearance of an enhanced $S U(n)$ gauge symmetry. It is natural to ask what happens to other enhanced gauge symmetries such as $E_{8}$ and how it is consistent with the global picture of the Dmanifold we have developed above.

Let us consider this in a little more detail. The local singularity of $E_{8}$ is given by

$$
x^{5}+y^{3}+z^{2}=\mu
$$

If we deform by a polynomial in $y$ we get two $A_{4}$ singularities each of which corresponds to 57 -branes of one $(p, q)$ string coming together. So we can predict two $S U(5)$ gauge symmetries. If we deform by a polynomial in $x$ we get four $A_{2}$ singularities each of which corresponds to 37 -branes of some $\left(p^{\prime}, q^{\prime}\right)$ string coming together. So again we can predict four $S U(3)$ gauge symmetries. If we do not deform at all we cannot predict what gauge symmetry we have because we have a region where there is no $(p, q)$ string in which the coupling is weak. In this way we avoid the contradiction of having to see an $E_{8}$ gauge symmetry in terms of $D$-branes. This is somewhat analogous to the avoidance of similar contradictions for type I-heterotic duality [23].

Here we have proposed that heterotic theory compactified on $T^{2}$ has a strong coupling regime which behaves like a 10 dimensional type IIB string with some 7-branes. Note however that if we wish to describe it in terms of a weak coupling limit of some string theory we will at most get an $A_{n}$ type gauge symmetry. On the other hand type I-heterotic duality similarly suggests a decompactified type I string [20], which are somewhat analogous to the case at hand except that in such cases the weak coupling regime will give only a $D_{n}$ type gauge symmetry.

2 Note also that we can also deduce that if we compactify only on one $S^{1}$ and use T-duality on that circle we have a consistent background of type IIA with 24 6-branes, which is equivalent to $\mathrm{M}$ theory on K3. 


\section{Type IIB and F-theory}

In the above 8 dimensional example we have seen how crucial the modulus $\tau$ has been in constructing a solution and in some sense it is convenient to think of it as describing the complex moduli of a $T^{2}$, in which case we can think of the above 8 dimensional solution as 'compactification' from 12 dimensions to 8 dimensions, on a $K 3$. Of course this $K 3$ has to admit an elliptic fibration with a frozen Kahler class for the torus. With the lesson of string dualities in geometrizing various symmetries the idea of a 'real' $T^{2}$ is very appealing. In fact this can be partially realized as follows [1] [6]: Consider M-Theory on $T^{2}$. This theory has an $S L(2, \mathbf{Z})$ symmetry as a geometric symmetry of the torus. It also has a scale corresponding to the area of $T^{2}$. If we take this are to zero, and use the relation between M-theory and type IIA, and use T-duality to relate to type IIB one can see that we will end up with type IIB theory in 10 dimensions in this limit. Even though this partially geometrizes the $S L(2, \mathbf{Z})$ of type IIB, the zero area limit we are taking is singular and in this limit the geometric description of M-theory breaks down. One is left with a desire to explain this $S L(2, \mathbf{Z})$ in a more direct fashion on the type IIB side. One would think that there should be a dual to type IIB, just as M-theory is dual to type IIA.

In fact we will see now that one can use the strong/weak duality above to derive the existence of a 12 dimensional theory with signature $(10,2)$. The strong/weak duality of type IIB, together with the observation of Polchinski [24] that D-branes carry RR charge, implies that the dual of fundamental type IIB string which couples to $B_{\mu \nu}^{A}$ is a Dirichlet 1brane, the D-string, which couples to $B_{\mu \nu}^{P}$. The modes on the Dirichlet 1-brane worldsheet are the same as one expects for type II string theory, namely the dimensional reduction of $10 \mathrm{~d}$ YM to $2 \mathrm{~d}$. However, there is an extra mode: the $U(1)$ gauge field which lives on the worldbrane seems apriori to have no analog for the worldsheet of the type IIB string theory. Thus we are faced with quantizing a string with $N=1$ worldsheet supergravity together with $N=1$ super-Maxwell. This exact problem was in fact already considered a while back [8] in constructing heterotic versions of type II strings and $N=2$ strings, the relevant features of which we will now review. We will not need, however, the coupling of type II string to $N=2$ strings and shall mainly concentrate on a reformulation of type IIB strings.

If we introduce on the worldsheet of type IIB strings a $U(1)$ super-Maxwell field the critical dimension of the theory changes, because now we have to introduce additional ghosts of spin $(0,1)$ which shifts the central charge by -2 . So the critical dimension is 
now $10+2=12$. Moreover the addition of new ghosts implies that the signature of the additional space is $(1,1)$, giving the total space a signature of $(10,2)$. Consider $v^{\mu}$ a vector in the $(10,2)$ space. Then the $U(1)$ current couples to $v_{\mu} D X^{\mu}$. For BRST invariance it turns out that $v \cdot v=0$, i.e., $v$ is a null vector. Choose coordinates so that $v$ lies in a $(1,1)$ subspace $M^{1,1}$. The BRST invariance of the states implies a null reduction of the theory and thus the physical states carry no momentum or oscillation along $M^{1,1}$. Thus the physical states are the same as that of the type IIB theory. So this is consistent with D-string being dual to type IIB strings which until now lived in 10 dimensions. However we would like to take the existence of these two extra dimensions a bit more seriously as some kind of 'off-shell' states. For this purpose it is convenient to compactify the $M^{1,1}$ part of the space. If we compactify left- and right-movers independently we get a Narain lattice

$$
\Gamma_{L}^{1,1} \oplus \Gamma_{R}^{1,1}
$$

where the subscripts $L, R$ refer to left- and right-moving momenta. Of course this can more generally be deformed so that the lattice does not have a product structure between left and right. The most general lattice we will get in this way is a lattice

$$
\Gamma_{L}^{1,1} \oplus \Gamma_{R}^{1,1} \rightarrow \Gamma^{2,2}
$$

which is the same Narain lattice as one would get upon compactification on a Euclidean $T^{2}$. The main difference is that we are exchanging one left-moving string mode with one right-moving one in interpreting the momenta for the compactification of $(10,2)$ theory on a $(1,1)$ space. At any rate the moduli of a $\Gamma^{2,2}$ lattice, however we may wish to interpret the individual components, is parametrized by two complex parameters, which can be identified as the complex structure $\tau$ and the Kahler structure $\rho$ of a Euclidean torus. Even though the BRST invariance rules out non-zero momentum states in the internal torus, the zero modes are not ruled out. We conjecture that the zero mode corresponding to one of the two moduli, say $\tau$ is physical and is to be identified with the $(\phi, \tilde{\phi})$ of type IIB. The fact that only one of the two moduli is physical is not so strange and is the case for $N=2$ strings where only one of the two moduli is dynamical. For example $N=2$ string amplitudes on $T^{2} \times R^{2}$ depends only on $\tau$ and not on $\rho$ [25]. This suggests that even though we are dealing with an internal theory with signature $(1,1)$ we can view it geometrically as if we are compactifying it on a Euclidean $T^{2}$ whose only dynamical degree 
of freedom is its complex structure. This is the version of F-theory we will be mostly using in this paper.

To make the above idea more concrete one will need to recall certain facts about $N=2$ strings. In the context of $N=2$ strings, it was proposed [8] that there should be a more symmetrical reformulation of the theory where the fundamental degree of freedom instead of strings with a $(1,1)$ worldsheet is an object with 4 dimensional worldvolume with signature $(2,2)$. Since the same reasoning applies here let us briefly review the idea of [8]. $N=2$ strings has a 4 dimensional target, but surprisingly has only one particle in its spectrum. In particular the infinitely many oscillatory modes of strings are not physical states. This is a bit strange because worldsheet is 2 dimensional and the target is 4 . What happens to the normal oscillations? Technically they are gotten rid of by the contributions of $U(1)$ current in the BRST invariance of physical states. This suggests that there may be another formulation where the worldvolume is 4 dimensional in which case the absence of oscillatory modes will be more manifest. In fact if we just view string as a 'geometrization' of Virasoro algebra, we will also be led to the same conclusion. From the algebraic point of view the coordinates of a Riemann surface arises by considering evolution operator

$$
z^{L_{0}}
$$

Similarly in the $N=1$ super-Virasoro we add an odd variable $\theta$ and consider the evolution operator

$$
z^{L_{0}} \exp \left(\theta G_{0}\right)
$$

the $(z, \theta)$ is then geometrically interpreted as the coordinates of a super-Riemann surface. Now, if we have a Virasoro algebra together with a $U(1)$ current, then we need two even coordinates $(z, u)$ in terms of which the evolution operator is given by

$$
z^{L_{0}} u^{J_{0}}
$$

Thus we are naturally led to a 4 dimensional worldvolume theory. Moreover in the $N=2$ string the signature is dictated by the fact that the target has signature $(2,2)$.

In the case at hand we also have a $U(1)$ current algebra and the geometrization naturally suggests a $(2,2)$ reformulation of the theory. This will also 'explain' why we have no oscillatory modes in the compactified $(1,1)$ part of the $(10,2)$ space. To connect with the usual $(9,1)$ reformulation it is natural to believe that the wrapping of a $(1,1)$ part of the $(2,2)$ worldvolume of the F-theory about the compact $(1,1)$ space, leaves us 
with a $(1,1)$ string in 10 dimensions, to be identified with type IIB strings. In such a picture $(p, q)$ strings [1] may arise from distinct wrappings of the $(1,1)$ part of the $(2,2)$ worldvolume around the $(1,1)$ compact space. It seems plausible that the description of this $(2,2)$ worldvolume theory is related to some of the supersymmetric self-dual theories proposed in [26] [27]. Also a prediction of F-theory (and more precisely the type II string with an extra $U(1)$ on worldsheet) is the existence of a peculiar not fully lorentz invariant supergravity theory in $(10,2)$ dimensions, whose formulation requires the choice of a null vector-it will be interesting to develop this further.

The idea of a 12 dimensional theory underlying type IIB has been considered by other physicists: It was pointed out to us by Sezgin that, quite independently from $N=2$ string considerations [8] reviewed above, it had also been conjectured based on p-brane/supersymmetry considerations [28]. We have been told (by Banks and Seiberg) that the idea of a $(10,2)$ theory underlying the type IIB theory has also been recently considered [29]. Also a 12 dimensional Euclidean origin for type IIB was hinted at recently in [30] though we do not understand the relation of those considerations to the ones in the present paper.

\section{Compactifications of F-Theory to Lower Dimensions}

The idea of considering lower dimensional compactifications of F-theory is a simple generalization of the compactification down to eight dimensions discussed in section 2 . In reformulating what we found in that case in terms of F-theory, we should say that we have a compactification of F-theory on $K 3$ manifolds which admit elliptic fibration and where only the complex moduli of the elliptic fiber is dynamical. Moreover we have to restrict the moduli so that we have a fixed $S^{2}$ sitting in $K 3$. In this reformulation the fact that we got 18 complex moduli together with one overall Kahler class of $S^{2}$ is a well known mathematical fact for elliptically fibered $K 3$ surface.

Now consider a manifold $K$ which admits an elliptic fibration. We can then consider compactifications of F-theory on the manifold $K$. If we compactify further on $K \times S^{1}$ this theory is on the same moduli as M-theory compactified on $K$, at least if as in the discussion of section 2, the adiabatic argument can be trusted. This relation with the M-theory will be useful in determining certain general features of the F-theory compactification, such as the number of supersymmetries and the massless spectrum. In particular this relation 
motivates consideration of compactifications of F-theory on manifolds which preserve a covariantly constant spinor.

Note that upon a further compactification on $S^{1}$ we get the relation between type IIA compactification and M-theory. So we conclude that type IIA compactification on $K$ is on the same moduli as M-theory on $K \times S^{1}$ which is on the same moduli as F-theory on $K \times S^{1} \times S^{1}$. This structure is useful to keep in mind as we briefly discuss certain compactifications of F-theory below.

We will now briefly consider the compactifications of F-theory on Calabi-Yau threefolds, $G_{2}$ holonomy and $\operatorname{Spin}(7)$ holonomy manifolds to 6,5 and 4 dimensions respectively.

\subsection{Compactification to $6 d$}

If we consider compactifications of F-theory on Calabi-Yau we end up with a theory in 6 dimensions with $N=1$. To see the number of supersymmetries note that compactifying further on $T^{2}$ we should get $N=2$ in four dimensions, as that would correspond to ordinary compactifications of type IIA on Calabi-Yau manifolds. This gives us a wealth of new models as there are a large number of Calabi-Yau manifolds which admit elliptic fibrations! We are currently analyzing these models [31]. Some of these models descend down in four dimensions to the heterotic/type II dualities found in 4 dimensions [32], and can be viewed as duals of the corresponding heterotic string compactifications on $K 3$, i.e., a duality in 6-dimensions. This is in line with the eight dimensional example discussed in section 2, where heterotic/type II duality in 6 dimension led to heterotic/F-theory duality in 8 dimensions. Other examples will have no analog heterotic duals but may have interesting type I duals.

Consider an elliptic Calabi-Yau $K$ with hodge numbers $h^{1,1}$ and $h^{2,1}$. Let us ask what is the spectrum of the $N=1$ theory in 6 dimensions we will end up with. In 4 dimensional terms we have $h^{2,1}+1$ hypermultiplets and $h^{1,1}$ vector multiplets. Apart from the $N=1$ supergravity multiplet in 6 dimensions we have tensor multiplets, vector multiplets and hypermultiplets. Let us denote the number of tensor multiplets by $T$, vector multiplets by $V$ and hypermultiplets by $H$. Note that upon dimensional reduction to $d=4$, vector and tensor multiplets convert to vector multiplets of $N=2$, and the hypermultiplets remain hypermultiplets. The $d=6, N=1$ supergravity multiplet leads, in addition to $N=2$ supergravity multiplet, to 2 vector multiplets in $d=4$. We thus learn that $V+T=h^{1,1}-2$, $H=h^{2,1}+1$. We can also determine what $T$ and $V$ are separately. The Calabi-Yau $K$ has $h^{2,1}$ complex deformations. This fact remains true even if we restrict them to the ones 
admitting elliptic fibrations [33]. Together with some other modes (including $A^{+}$constant modes) they make up $h^{2,1}$ of the hypermultiplets expected. Let $k$ denote the number of Kahler deformations of the Calabi-Yau $K$, which does not change the Kahler class of the elliptic fiber. Then we get $k$ scalars corresponding to the Kahler classes. Note that just as in the eight dimensional example discussed in section 2 the scalars are not complexified because the $B$-fields are frozen to be zero. Out of these $k$ scalars, the one corresponding to the overall volume of the four manifold which is the base of the elliptic fibration, together with certain modes (including the $A^{+}$field coming from the volume of the four manifold) form a hypermultiplet, giving a total of $h^{2,1}+1$ hypermultiplets accounted for. The other $k-1$ scalars are part of $k-1$ tensor multiplets (note that a tensor multiplet has only one scalar consistent with the freezing of the $B$-fields). We thus have

$$
T=k-1 \quad V=h^{1,1}-k-1
$$

Note that as we vary the moduli and as some of the 7-branes coincide we end up with an enhanced gauge symmetry as is by now familiar.

To make the discussion a little more concrete let us consider an example (which as compactification to four dimensions was also considered in the context of D-manifolds in [7] and has been extensively studied in [34] ). Consider F-theory on an elliptic Calabi-Yau where it has two elliptic fibers over $S^{2}$. The complex modulus of the first torus is denoted by $\tau_{1}$ and is identified with the type IIB complex coupling constant in 10 dimensions. We denote the complex modulus of the other torus by $\tau_{2}$. Consider a fibration over $S^{2}$ where $S^{2}$ wraps 12 times over each of the two fundamental domains. We can take the two fibrations determined by

$$
y_{i}^{2}=x_{i}^{3}+f_{i}^{4}(z) x_{i}+f_{i}^{6}(z)
$$

where $i=1,2$ corresponds to the two tori. The corresponding Calabi-Yau has hodge numbers $h^{1,1}=19, h^{2,1}=19$. The above fibration describes 19 complex parameters: 24 parameters from the coefficients of the polynomials, minus 3 from $S L(2)$ and 2 from independent rescalings which does not affect the tori, leaving us with $24-3-2=19$. In this case the number of Kahler parameters, consistent with preserving one elliptic curve fibered is $k=10$. So from above discussion we have $T=9$ tensor multiplets and $V=8$ vector multiplets3. Note that we could have deduced $V=8$ by noting that the independent

3 The fact that from geometrical fibrations of the second torus we get tensor multiplets can also be seen by the fact that type IIB near an $A_{n-1}$ singularity is dual to type IIA with $n$-symmetric fivebranes [21], which gives rise to tensor multiplets on the fivebrane worldvolume [35]. 
complex parameters in the first torus is 12 minus 3 from $S L(2)$ and 1 from overall rescaling, giving a total of 8 independent 7-brane charges 1 .

\subsection{Compactification to $d=5$}

Compactification of F-theory on an elliptic $G_{2}$ holonomy 7 -manifold leads to a theory in 5 dimensions which has $N=1$ supersymmetry. These theories will appear in the $d=$ 4, $N=1$ moduli of M-theory compactification on $G_{2}$ holonomy manifolds to 4 dimensions. It would be interesting to study them from this point of view. Note that the existence of elliptic $G_{2}$ and elliptic $\operatorname{Spin}(7)$ manifolds has been established in [37. In fact many of the examples constructed by Joyce admit elliptic fibration.

\subsection{Compactification to $d=4$}

This is perhaps the most interesting case to consider. We compactify F-theory on an elliptic Spin(7) holonomy manifold. We immediately run to a puzzle: By the general arguments above upon a further $S^{1}$ compactification to three dimensions this should be on the same moduli space as M-theory on $\operatorname{Spin}(7)$ manifolds, which has $N=1$ supersymmetry in three dimensions. However this implies that the original theory in four dimensions cannot have $N=1$ because that would have led to $N=2$ in three dimensions. So the puzzle seems to be that we are predicting the existence of a theory in four dimensions which has no supersymmetry but upon compactification on $S^{1}$ in the limit of small circle it develops an $N=1$ supersymmetry in three dimensions! This puzzle is resolved by Witten's observation [9] that $N=1$ supersymmetric theories in $d=3$ do not exhibit a supersymmetric spectrum and in particular there would be no obstruction to being connected to a four dimensional theory with no manifest supersymmetry. In fact it was proposed in [9] that if a supersymmetric theory in three dimensions is connected to a nonsupersymmetric theory in four dimensions this may lead to a resolution of the cosmological constant problem. What was not clear up to now is whether this beautiful idea admits a concrete physical realization. Here we see that not only this idea can be realized but that it is generically the case for compactifications of M-theory on elliptic Spin(7) holonomy

4 Just as we were about to release this paper, a paper appeared [36] which describes an $N=1$ model with the same spectrum as the model discussed above. It is quite plausible that the two models are dual to one another. This is supported further by the fact that both models appear to have the same enhanced gauge symmetries. 
manifolds. Type IIA string compactified to 2 dimensions on an elliptic Spin(7) holonomy manifold is on the same moduli as M-theory compactified on the same manifold to 3 dimensions which is also on the same moduli as F-theory compactified on the same manifold to 4 dimensions (it is quite amusing that at least the uncompactified dimensions (if not the signature) in each of these three cases agrees with the dimension of worldvolume of the corresponding theory). Note that for compactifications of type IIA on an eight manifold we have a term generated [38] which should be canceled by turning on appropriate fields (such as by turning on string condensates in the type IIA case or membranes in the Mtheory case [39]). It thus seems that compactifications of strings, M-theory and F-theory to 2,3 and 4 dimensions on elliptic $\operatorname{Spin}(7)$ holonomy manifolds should be studied very intensively and may hold the key to connecting string theory to the real world.

This work was done while I was visiting Rutgers University Physics department. I greatly benefited from the stimulating research environment there. In particular I would like to thank T. Banks and N. Seiberg for participation at the initial stages of this work and for making many helpful suggestions. In addition I am grateful to D. Morrison for explaining to me various mathematical facts about elliptically fibered manifolds. I would also like to thank M. Bershadsky, H. Ooguri, V. Sadov and E. Sezgin for valuable discussions. This research was supported in part by NSF grant PHY-92-18167. 


\section{References}

[1] J. Schwarz, hep-th/9508143, hep-th/9510086

[2] P. Horava and E. Witten, hep-th/9510209

[3] K. Dasgupta and S. Mukhi, hep-th/9512196

[4] E. Witten, hep-th/9512219

[5] M.J. Duff, R. Minasian and E. Witten, hep-th/9601036

[6] P.S. Aspinwall, hep-th/9508154.

[7] M. Bershadsky, V. Sadov and C. Vafa, hep-th/9510225

[8] H. Ooguri and C. Vafa, Mod. Phys. Lett. A5 (1990) 1389; Nucl. Phys. B361 (1991) 469; Nucl. Phys. B367 (1991) 83.

[9] E. Witten, Int. J. Modern Phys. A10 (1995) 1247; hep-th/9506101

[10] C.M. Hull and P.K. Townsend, Nucl. Phys. B438 (1995) 109

[11] B. R. Greene, A. Shapere, C. Vafa and S.-T. Yau, Nucl. Phys. B337 (1990) 1

[12] G. Gibbons, M.B. Green and M.J. Perry, hep-th/9511080

[13] J. Dai, R. G. Leigh and J. Polchinski, Mod. Phys. lett, A4 (1989) 2073

[14] P. Horava, Phys. Lett. B231 (1989) 251

[15] M. Bershadsky, V. Sadov and C. Vafa, hep-th/9511222

[16] M. Li, hep-th/9510161

[17] C.G. Callan, C. Lovelace, C.R. Nappi and S.A. Yost, Nucl. Phys. B308 (1988) 221

[18] M. Douglas, hep-th/9512077

[19] C. Vafa and E. Witten, hep-th/9507050

[20] E. Witten, hep-th/9503124

[21] H. Ooguri and C. Vafa, hep-th/9511164

[22] C. Vafa, unpublished.

[23] J. Polchinski and E. Witten, hep-th/9510169

[24] J. Polchinski, hep-th/9510017

[25] H. Ooguri and C. Vafa, hep-th/9505183

[26] W. Siegel, Phys. Rev. Lett. 69 (1992) 1493.

[27] E. Bergshoeff and E. Sezgin, Phys. Lett. B292 (1992) 87.

[28] M. Blencowe and M. Duff, Nucl. Phys. B310(1988) 387

[29] P. Townsend et. al., unpublished

[30] C. Hull, hep-th/9512181

[31] D. Morrison and C. Vafa, work in progress.

[32] S. Kachru and C. Vafa, Nucl. Phys. B450 (1995) 69

[33] D. Morrison, private communication.

[34] C. Schoen, J. fur Math. 364 (1986) 85.

[35] C.G. Callan, J.A. Harvey and A. Strominger, Nucl. Phys. B359 (1991) 611.

[36] A. Sen, hep-th/9602010 
[37] D.D. Joyce, Compact Riemannian 7-manifolds with Holonomy $G_{2}$, to appear in J. Diff. Geom.; Compact Riemannian 8-manifolds with Holonomy Spin(7) to appear in Inv. Math.

[38] C. Vafa and E. Witten, Nucl. Phys. B447 (1995) 261

[39] C. Vafa and E. Witten, unpublished 